\title{
Charged kaon multiplicities in semi-inclusive deep-inelastic scattering from COMPASS
}

\author{
E. Seder* \\ On behalf of the COMPASS Collaboration \\ CEA Saclay, DSM/IRFU/SPhN \\ F91191 Gif-sur-Yvette, France \\ E-mail: erin.sederecern.ch
}

\begin{abstract}
The latest measurements of $K^{ \pm}$multiplicities in semi-inclusive deep-inelastic scattering from the COMPASS experiment at CERN are presented. The data were collected using a $160 \mathrm{GeV}$ muon beam incident on an isoscalar ${ }^{6} \mathrm{LiD}$ target. The large statistics collected cover a wide kinematic range: $1<Q^{2}<50(\mathrm{GeV} / c)^{2}, 0.1<y<0.7,0.004<x<0.4$, and $0.2<z<0.85$. Quark fragmentation functions extracted from a leading order fit to the COMPASS data give $D_{\text {fav }}^{K}$ and $D_{u n f}^{K}$, both of which are significantly higher than DSS results. We present also the sum of $K^{+}$and $K^{-}$multiplicities integrated over $z$. The results of the sum suggests that ratio of fragmentation functions $D_{s t r} / D_{\text {fav }}$ is lower than current DSS expectations.
\end{abstract}

XXIII International Workshop on Deep-Inelastic Scattering,

27 April - May 12015

Dallas, Texas

\footnotetext{
* Speaker.
} 


\section{Introduction}

Extensive efforts have been made to understand the partonic structure of the nucleon through determination of non-perturbative Parton Distribution Functions (PDFs). Currently of particular interest is the strange quark sector of the nucleon, for which both the unpolarised, $s(x)$, and polarised, $\Delta s(x)$, PDFs are still poorly known. The strange quark PDFs can be accessed through measurements of semi-inclusive lepton-nucleon deep inelastic scattering (SIDIS). However, recent analysis [1] has shown that a good determination of $\Delta s(x)$ from SIDIS relies on the thorough understanding of the hadronisation mechanisms, encoded in non-perturbative Fragmentation Functions (FFs), which describe the transition from final-state partons into colour-neutral hadronic bound states. Determination of strange FFs remain at a very preliminary stage of knowledge with a growing interest in more accurate and precise measurements.

To study strange quark FFs in SIDIS, the relevant observable is the kaon multiplicity, $M^{K}$, defined as the differential cross section for kaon production normalised to the inclusive DIS cross section. The relevant kinematic variables are the Bjorken scaling variable, $x$, the virtual photon transfer four-momentum squared, $Q^{2}$, and the virtual photon energy fraction carried by final-state kaon, $z$. At leading order (LO) in pQCD, $M^{K}$ can be written as:

$$
\frac{\mathrm{d} M^{K}\left(x, z, Q^{2}\right)}{\mathrm{d} z}=\frac{\mathrm{d}^{3} \sigma^{K}\left(x, z, Q^{2}\right) / \mathrm{d} x \mathrm{~d} Q^{2} \mathrm{~d} z}{\mathrm{~d}^{2} \sigma^{D I S}\left(x, Q^{2}\right) / d x d Q^{2}}=\frac{\sum_{q} e_{q}^{2} q\left(x, Q^{2}\right) D_{q}^{K}\left(z, Q^{2}\right)}{\sum_{q} e_{q}^{2} q\left(x, Q^{2}\right)}
$$

where $q\left(x, Q^{2}\right)$ is the quark PDF for the flavour $q$, and $D_{q}^{K}\left(z, Q^{2}\right)$ the FF of quark flavor $q$ into kaons.

New precise SIDIS measurements of particles carrying strangeness (kaons) from experiments where kaons are copiously produced and well identified, as is the case of the work presented here, greatly expand on the limited current SIDIS measurements available, allowing for better parameterisation of strange quark FFs.

\section{Data analysis}

The multiplicities $M^{\mathrm{K}^{ \pm}}$are calculated in bins $x, y$ (the lepton energy fraction carried by the virtual photon), and $z$. The choice of the $z$ and $x$ variables is natural because multiplicities depend most strongly on these variables. The third variable is chosen to be $y$, since it is close to a laboratory variable, and thus it is well suited for the acceptance evaluation. It is preferred to $Q^{2}$, which is strongly correlated to $x$ in COMPASS fixed target kinematics. The multidimensional binning used for the multiplicity extraction includes 9 bins in $\mathrm{x}$ and 5 bins in $\mathrm{y}$, as shown in Fig. 1, as well as 12 bins in $\mathrm{z}$.

The expression of the multiplicities, $M^{\mathrm{K}^{ \pm}}$, is given by the kaon yield $N^{\mathrm{K}^{ \pm}}$in $(x, y, z)$ bins divided by the number of DIS events $N^{\text {DIS }}$ in $(x, y)$ bins, corrected by acceptance $A^{\mathrm{K}^{ \pm}}$and diffractive vector meson contamination $B^{\mathrm{K}^{ \pm}}$:

$$
\frac{\mathrm{d} M^{\mathrm{K}^{ \pm}}(x, y, z)}{\mathrm{d} z}=\frac{1}{N^{\operatorname{DIS}}(x, y)} \frac{\mathrm{d} N^{\mathrm{K}^{ \pm}}(x, y, z)}{\mathrm{d} z} \frac{B^{\mathrm{K}^{ \pm}}(x, y, z)}{A^{\mathrm{K}^{ \pm}}(x, y, z)}
$$




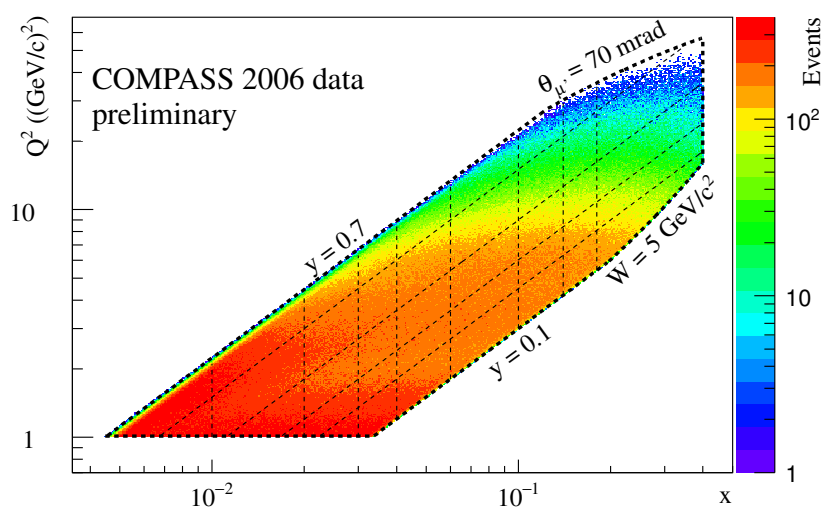

Figure 1: Kinematic variables for DIS events, $Q^{2}$ vs $x$. Dashed lines indicate the $\mathrm{x}$ and y bin limits.

\subsection{Acceptance correction}

The acceptance correction, $A^{\mathrm{K}^{ \pm}}$, takes into account the limited geometric and kinematic acceptance of the spectrometer and the efficiency of event reconstruction. It is evaluated using a Monte-Carlo (MC) simulation of muon-nucleon deep inelastic scattering processes. Events are generated with the LEPTO [2] generator where the parton hadronisation mechanism is simulated using the JETSET package [3] with the tuning from ref. [4]. To avoid introducing a strong dependence on the event generator used in the simulation, the extraction of kaon multiplicities from the $\mathrm{MC}$ is performed in narrow kinematic bins of $x, y$ and $z$. The geometry of the spectrometer is simulated using the GEANT3 toolkits [5], and the MC data are reconstructed with the same software as the experimental data [6] taking detector efficiencies and resolutions into account. The acceptance is calculated from the ratio of the reconstructed multiplicities over generated multiplicities where the reconstructed events and particles are subject to the same kinematic and geometric cuts as the data, while the generated ones are subject to kinematic requirements only.

\subsection{Diffractive vector meson contamination}

Usually it is assumed that hadrons produced in SIDIS originate from lepton-parton scattering. However, the scattering of a lepton off a nucleon can also result in the diffractive production of vector mesons (DVM). These particles decay into lighter mesons that cannot be distinguished from the one resulting from the hadronisation of a quark originating from the target nucleon. The diffractive vector meson events can also lead to a contamination in DIS events. In this work, the two channels studied are diffractive $\phi$ and $\rho^{0}$. As mesons stemming from DVM decay cannot be separated from the ones resulting from SIDIS, the evaluation of their contribution to the multiplicities is estimated using MC. Separate cross section normalised MC samples are used, based on different generators (SIDIS using LEPTO [2], and DVM events using HEPGEN [7]) and the same event reconstruction chain as described in the previous section is used. The fraction of DVM is calculated in the same binning as the multiplicities, and the resulting correction, $B^{\mathrm{K}^{ \pm}}$, is applied to the measured multiplicities as a multiplicative factor. 


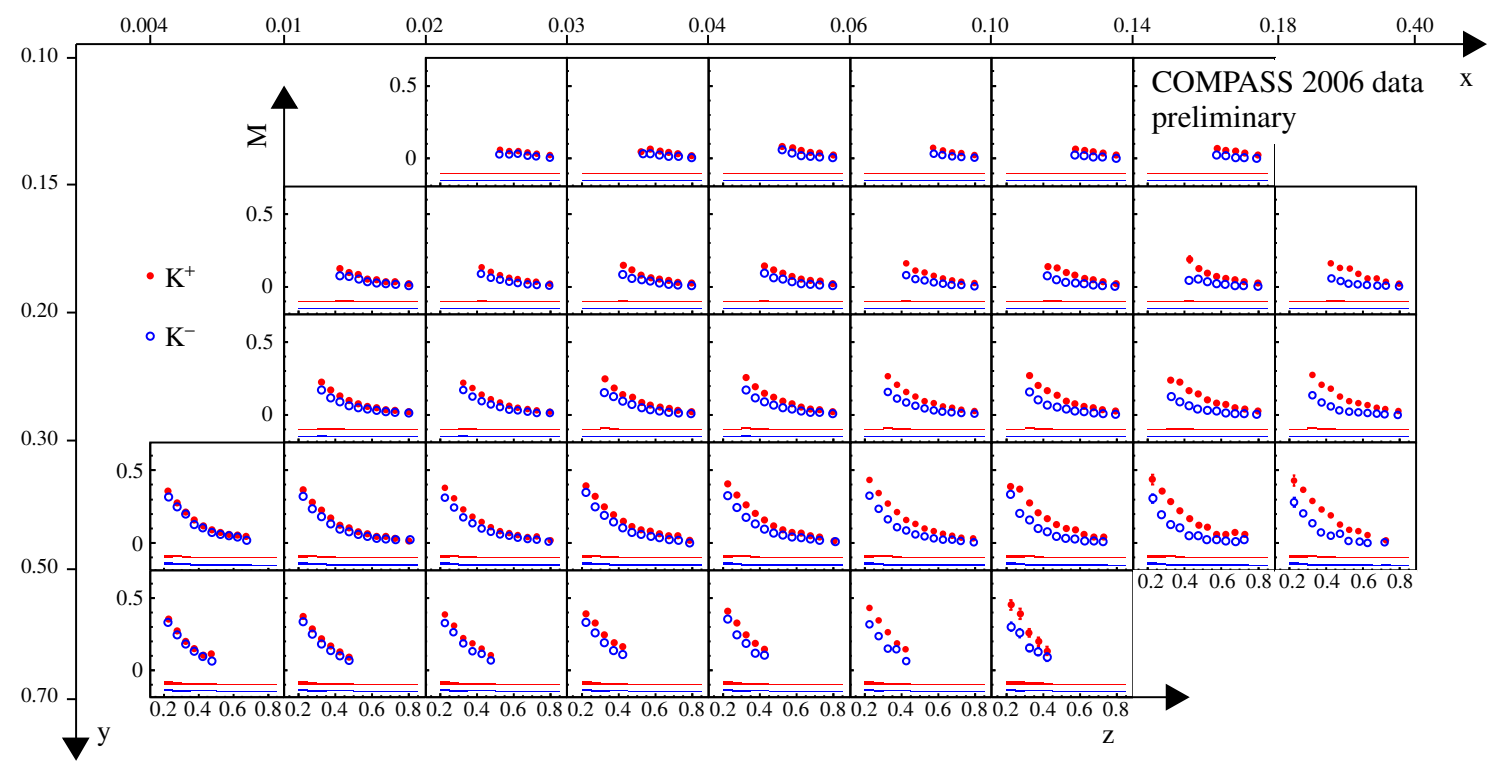

Figure 2: Final multiplicities as a function of $z$ for $(x, y)$ bins. The red markers correspond to positive kaons and the blue markers to negative kaons. Both diffractive vector meson and acceptance corrections have been applied. The asymmetric systematic errors for radiative correction (100\% of the effect) on kaons do not extend above the markers, i.e. the systematics related to the radiative correction are not shown. This error averages $\approx 5 \%$ at large $\mathrm{x}$, up to $\approx 11 \%$ at lower $\mathrm{x}$, high $\mathrm{y}$. All other systematic errors are shown as bands.

\subsection{Radiative effects}

The experimental multiplicities are affected by QED radiative effects, which introduces a systematic bias of the observed (measured) kinematics with respect to the true kinematics. The most important contributions at first order are the initial and final state radiation of a real photon by the incoming and outgoing lepton. The correction factor used to take into account these phenomena is defined versus $x$ and $y$, calculated using the Mo and Tsai scheme [8] taking into account the target composition. The bias on the $\mu$ kinematics upon real photon emission affects in turn the reconstruction of the hadron energy fraction $z$. This effect is not taken into account thus far and is still under investigation. A very conservative systematic error equalling $100 \%$ of the correction on kaons is applied to the preliminary results.

\section{Results}

The final multiplicities with all corrections are shown in a multidimensional binning in $x, y$ and $z$ ( 9 columns, 6 rows, 12 points, respectively) in Fig. 2. Bins with less than $30 \%$ acceptance have been excluded from the analysis and are not shown. The data show a strong $z$ dependence with a much weaker dependence in $x$ and $y$. As expected due to u quark dominance $M^{\mathrm{K}^{+}}$is greater than $M^{\mathrm{K}^{-}}$.

\subsection{Extraction of quark fragmentation functions (FFs) into kaons from a LO QCD fit of kaon multiplicities}

The extraction of FFs from the measured $K^{ \pm}$multiplicities is performed in a LO pQCD ap- 
proach according to Eq. 1.1 , using a standard $\chi^{2}$ minimisation over the data points, where

$$
\chi^{2}=\sum_{i, j, k} \frac{\left(T\left(x_{i}, z_{j}, Q_{k}^{2}\right)-M\left(x_{i}, z_{j}, Q_{k}^{2}\right)\right)^{2}}{\sigma^{2}\left(x_{i}, z_{j}, Q_{k}^{2}\right)}
$$

$M$ are the measured multiplicities and $\sigma$ the statistical errors. $T$ are multiplicities evaluated according to equation 1.1 for a set of parameters for given parametrisations. Due to the large $Q^{2}$ span of the data, the $T_{j}$ are evaluated at $Q_{0}^{2}=1 \mathrm{GeV}^{2} / c^{2}$ and evolved to their actual $Q_{j}^{2}$ using a DGLAP $Q^{2}$ evolution code provided by M. Hirai and S. Kumano[9].

For the combined analysis of $K^{+}$and $K^{-}$multiplicities, isospin and charge symmetry are imposed. This yields to the three FFs :

$$
\begin{aligned}
D_{\mathrm{fav}}^{K} & =D_{u}^{K^{+}}=D_{\bar{u}}^{K^{-}} \\
D_{\mathrm{str}}^{K} & =D_{\bar{s}}^{K^{+}}=D_{s}^{K^{-}} \\
D_{\mathrm{unf}}^{K}=D_{\bar{u}}^{K^{+}}=D_{s}^{K^{+}} & =D_{u}^{K^{-}}=D_{\bar{s}}^{K^{-}}=D_{\bar{d}}^{K^{ \pm}}=D_{d}^{K^{ \pm}}
\end{aligned}
$$

For this analysis, the following parametrisation is used :

$$
\begin{array}{r}
z D_{i}\left(z, Q_{0}^{2}\right)=\frac{N_{i} z^{\alpha_{i}}(1-z)^{\beta_{i}}\left(1+\gamma_{i}(1-z)^{\delta_{i}}\right)}{\int_{0.2}^{0.85} d z z^{\alpha_{i}}(1-z)^{\beta_{i}}\left(1+\gamma_{i}(1-z)^{\delta_{i}}\right)}, i=\{\text { fav }\} \\
z D_{j}\left(z, Q_{0}^{2}\right)=\frac{N_{j} z^{\alpha_{j}}(1-z)^{\beta_{j}}}{\int_{0.2}^{0.85} d z z^{\alpha_{j}}(1-z)^{\beta_{j}}}, j=\{\text { str, unf }, \text { glu }\}
\end{array}
$$

and the PDFs set from MSTW08 in LO [10] was chosen.

To determine the uncertainties, both statistical and systematical, of the extracted FFs, the replica method [11] is used. The results obtained for $D_{\text {fav }}^{K}$ and $D_{\text {unf }}^{K}$ as a function of z are shown in Fig. 3. The values obtained are significantly above the DSS results (LO and NLO) for both cases (favoured and unfavoured). At this stage of analysis, the result for $D_{\text {str }}^{K}$ is considered not well constrained (it depends strongly on the choice of initial parametrisation).
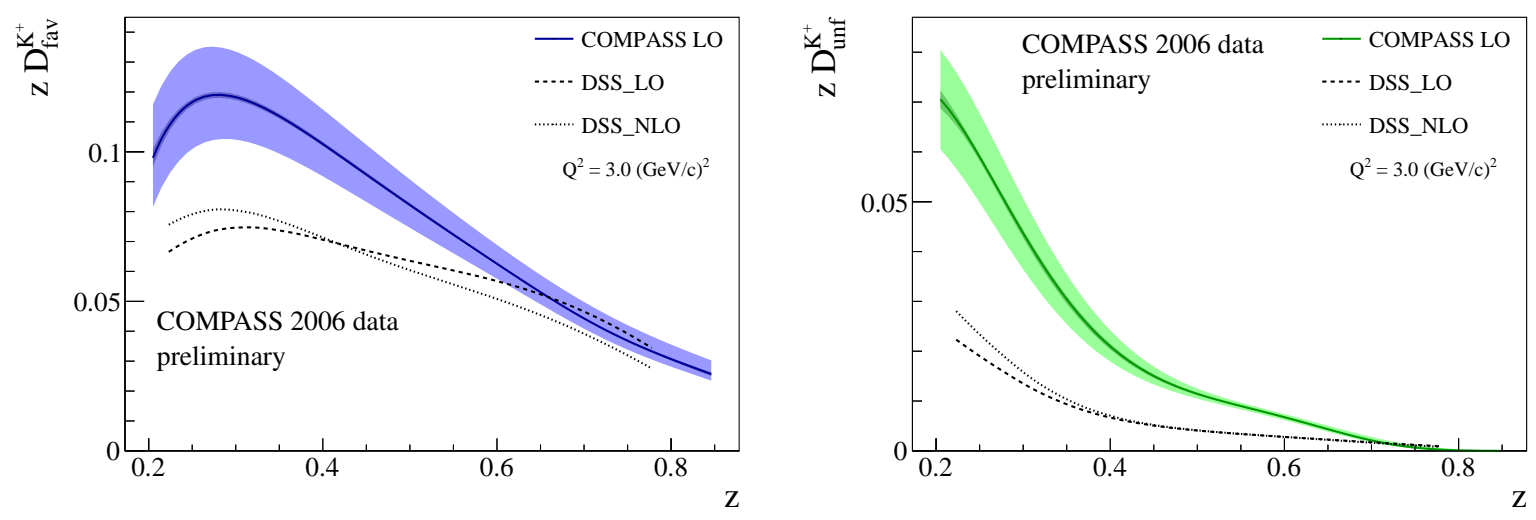

Figure 3: The favoured (left) and the unfavoured (right) quark FFs $z D(z)$ into kaons from the COMPASS LO fit compared to the DSS fit at LO and NLO. The fit is done based on the statistical errors only. The FF error bands correspond to statistical (darker band) and correlated systematic (lighter band) uncertainties 


\subsection{Sum of charged kaon multiplicities $M^{K^{+}}$and $M^{K^{-}}$}

One of the main goals of the kaon multiplicity analysis is the extraction of kaon strange fragmentation functions. These FFs allow for the extraction of the strange quark polarisation in the nucleon obtained from polarised SIDIS analyses. From this point of view, the sum of positive and negative charge kaon multiplicities integrated over $z$ is of special interest.

For the isoscalar target, when expressed at LO the sum has a very simple form:

$$
\frac{d N^{K}}{d N^{D I S}}=\frac{Q D_{Q}^{K}+S D_{S}^{K}}{5 Q+2 S}
$$

where, $Q=u+\bar{u}+d+\bar{d}$ and $S=s+\bar{s}, D_{Q}$ and $D_{S}^{K}$ are combination of FFs, which can be expressed as $D_{Q}^{K}=4 D_{f a v}^{K}+6 D_{u n f}^{K}$ and $D_{S}^{K}=2 D_{s t r}^{K}+2 D_{u n f}^{K}$.

The multiplicity sum is presented in Fig. 4. The data are integrated over $z(0.20$ to 0.85$)$ and averaged over $y$ ( 0.1 to 0.7$)$. Only the $8 x$ bins that have sufficient $z$ coverage subsist. Each point is plotted at the measured $Q^{2}$.

At high $x$, the strange content of the nucleon can be neglected, and in a good approximation the measured multiplicity is related to $D_{Q}^{K} / 5$. The measured multiplicity value at high $x$ is 0.14 , which corresponds to $D_{Q}^{K} \approx 0.70$. In contrast, the present expected value from DSS is about $D_{Q}^{K} \approx 0.43 \pm 0.04$.

As $D_{Q}^{K}$ is expected to have a rather weak $Q^{2}$ dependence (when integrated over $z$ ), the value at higher $x$ (corresponding to higher $Q^{2}$ for COMPASS kinematics) can be used for lower $x$ values (corresponding to lower $Q^{2}$ for COMPASS kinematics) to pin down the $S D_{S}^{K}$ value.

As it is expected from DSS that $D_{s t r}>D_{f a v}$, we would expect to observe a rise of the kaon multiplicity sum for lower $x$ values where the term $S D_{\text {str }}$ cannot be neglected. Contrary to this expectation, we do not observe a large increase of the kaon multiplicity sum for low $x$ values. This suggests that ratio of FFs $D_{s t r} / D_{\text {fav }}$ is lower than current DSS expectations ${ }^{1}$.

As can be clearly seen from Fig. 4, HERMES results [12] and COMPASS results are not compatible. Our multiplicities are above those of HERMES in the full $x$ range There is, however, a reasonable agreement between our observed $\mathrm{x}$-dependence and LEPTO/JETSET expectations. However, one has to add that the low $x$ behaviour of the kaon multiplicity sum can be modified by radiative corrections. These corrections are still under investigation and a systematic of $100 \%$ of the correction on kaons has been added to the multiplicities, represented in Fig. 4 by the asymmetric error band over the points.

\section{Summary and conclusions}

We have presented multiplicities of positive and negative charge kaons measured from SIDIS of muons off an isoscalar target at COMPASS. Data are shown in bins of $x, y$ and $z$. They cover the kinematic range $1<Q^{2}<50(\mathrm{GeV} / c)^{2}, 0.1<y<0.7,0.004<x<0.4$, and $0.2<z<0.85$. A leading order fit of the kaon multiplicities was used to extract the favored $D_{\mathrm{fav}}^{K}$ and unfavored $D_{\mathrm{unf}}^{K}$

\footnotetext{
${ }^{1}$ Formally in COMPASS we extract a product $S D_{S}^{K}$, thus our result can also mean that $S(x)$ is smaller than in MSTW08. On the other hand, some LHC results shows that $S(x)$ is not suppressed w.r.t. non strange sea i.e. it should be larger than what is obtained from MSTW08.
} 


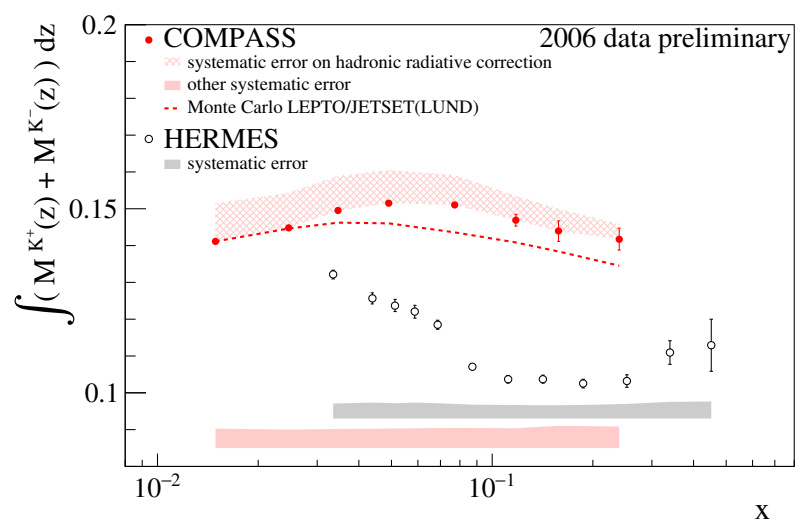

Figure 4: $M^{K^{+}}+M^{K^{-}}$versus x for COMPASS data (red points) averaged over $y(0.1$ to 0.7$)$ and integrated over $z(0.20$ to 0.85$)$ and for HERMES data [12] (black circles) integrated over $z(0.20$ to 0.80$)$. The asymmetric systematic error for radiative correction on kaons is shown point to point as a hatched band above the statistical error. All other systematic errors are shown in the full filled band. The dashed red line is the result obtained from the COMPASS Lund Monte Carlo.

quark FFs into kaons. The values obtained are significantly above the DSS results for both cases (favoured and unfavoured). The sum of positive and negative charge kaon multiplicities integrated over $z$ is also presented. Contrary to the expectation from DSS, we do not observe a large increase of the multiplicity sum for low $x$ values. This suggests that the ratio of FFs $D_{s t r} / D_{\text {fav }}$ is lower than current DSS expectations. Finally, compared to HERMES results, the multiplicity sum shows a significantly different $x$ dependence as well as an overall higher value.

\section{References}

[1] M. G. Alekseev et al. [COMPASS Collaboration], Phys. Lett. B 693 (2010) 227.

[2] G. Ingelman, A. Edin and J. Rathsman, Comput. Phys. Commun. 101 (1997) 108

[3] T. Sjostrand, hep-ph/9508391.

[4] C. Adolph et al. [COMPASS Collaboration], Phys. Lett. B 718 (2013) 922

[5] R. Brun, M. Caillat, M. Maire, G. N. Patrick and L. Urban, CERN-DD/85/1.

[6] P. Abbon et al. [COMPASS Collaboration], Nucl. Instrum. Meth. A 577 (2007) 455

[7] A. Sandacz and P. Sznajder, arXiv:1207.0333 [hep-ph].

[8] B. Badelek, D. Y. .Bardin, K. Kurek and C. Scholz, Z. Phys. C 66 (1995) 591.

[9] M. Hirai, S. Kumano, T.-H. Nagai, K. Sudoh arXiv:hep-ph/0702250v2 April 2007

[10] A.D. Martin, W.J. Stirling, R.S. Thorne, G. Watt, Eur. Phys. J.C 63 (2009)

[11] William H. Press, Saul A. Teukolsky, William T. Vetterling, Brian P. Flannery, Numerical Recipes in C (2Nd Ed.): The Art of Scientific Computing 1992

[12] A. Airapetian et al., Phys. Rev. D 89 (2014) 097101 